\title{
Synergism from combinations of infection-blocking malaria vaccines
}

\author{
Michael T White ${ }^{1 *}$ and David L Smith 2,3
}

\begin{abstract}
Plasmodium falciparum infections present novel challenges for vaccine development, including parasite replication dynamics not previously encountered for viral pathogens, and enormous diversity in target antigens. These challenges are illustrated by using a mathematical model to describe the association between the proportion of pre-erythrocytic or blood-stage parasites eliminated by vaccine-induced immune responses and the proportion of infections prevented. It is hypothesized that due to the requirement for all sporozoites to be eliminated to prevent infection, combining infection-blocking vaccines that confer protection through different biological mechanisms could lead to synergistic combinations of efficacy. Vaccines targeting blood-stage parasites may also combine synergistically if they combine to reduce the parasite multiplication rate to below the threshold of 1 .
\end{abstract}

Keywords: Malaria, Vaccine, Infection, Pre-erythrocytic, Synergy

\section{Background}

Plasmodium falciparum infections present novel challenges for vaccine development, including parasite replication dynamics not previously encountered for viruses, and enormous diversity in target antigens [1]. Malaria parasites express multiple life stages in humans, each presenting unique targets for vaccine-induced immune responses. Malaria infection can be prevented either by targeting the pre-erythrocytic (PE) stages and clearing sporozoites inoculated in the skin or infected hepatocytes in the liver, or by targeting blood-stage (BS) merozoites and infected red blood cells in the prepatent period after emergence from the liver and before parasite densities increase to detectable levels. The dynamics of these parasite life stages and corresponding immune kinetics also differ. Sporozoites pass from the skin to the liver without replicating, so clearing sporozoites or eliminating hepatocytes can prevent infection outright. The pass-through system also means that the immune system will be naturally exposed to and stimulated by relatively few sporozoites, but this number increases after repeated exposure to infectious bites of mosquitoes. BS parasites undergo periodic replication, so like viral

\footnotetext{
* Correspondence: m.white08@imperial.ac.uk

'Department of Infectious Disease Epidemiology, MRC Centre for Outbreak Analysis and Modelling, Imperial College, London, UK

Full list of author information is available at the end of the article
}

pathogens, the combined vaccine-induced and naturally acquired immune response can prevent infection if enough parasites are cleared so that the number of parasites is reduced from one generation to the next. Replication of BS parasites implies that the immune system will be exposed to very high parasite numbers from a single infection.

The sporozoite bottleneck would seem to make an easy vaccine target, but the potential for a single parasite to evade vaccine-induced immune responses and spark an infection makes the challenge of developing an effective PE vaccine deceptively difficult. The effect of BS malaria vaccines on BS parasites is qualitatively similar to successful vaccines against viruses, in that a threshold immunological correlate for sterile protection against infection is theoretically possible. Hepatic parasites that survive the PE immune response will release merozoites into the blood stream which invade and replicate within red blood cells, causing an increase in parasite numbers every two-day cycle by a factor defined as the parasite multiplication rate (PMR) [2]. PMR $>1$ will lead to an increase in parasite numbers and possibly an episode of clinical malaria, and PMR $<1$ will lead to a decrease in parasite numbers and the clearance of BS infection. BS vaccines prevent the replication of BS parasites by either preventing merozoites from invading red blood cells or clearing infected red blood cells. A BS vaccine that fails 
to reduce PMR $<1$ may still confer protection against clinical and severe episodes by providing time for the natural immune response to mature. In a study in Aotus monkeys, a reduction in median PMR from 15 to 5 in the early phase of infection was associated with subsequent clearance of parasites without developing symptoms of anaemia (Sandy Douglas - personal communication). In contrast to BS vaccines, which need not clear every parasite, $\mathrm{PE}$ vaccines must prevent the successful development of each sporozoite, a challenging prospect given that most evidence suggests that the number of sporozoites inoculated per infectious bite is highly skewed, with some bites injecting very large numbers of sporozoites [3].

\section{The potential benefits of multicomponent malaria vaccines}

Although both PE and BS vaccines induce immune responses that target individual parasites within the human host, the relationship between the average proportion of parasites killed and the proportion of infections prevented (efficacy against infection) is qualitatively different, as illustrated in Figure 1. Given that a single sporozoite evading the vaccine-induced immune response is sufficient to initiate malaria infection, and that during some mosquito bites large numbers of sporozoites may be inoculated, it is not predicted that there will be any threshold immunological correlate of protection at which sterile protection from infection is conferred, and hence that pre-erythrocytic malaria vaccines will be leaky [4]. This could explain, in part, why RTS,S has produced such high antibody responses, yet estimates of efficacy against infection are approximately 50\% [5]. The convex shape of the curve for $\mathrm{PE}$ vaccines in Figure 1a suggests that preventing $50 \%$ of infections requires that, on average, in excess of $90 \%$ of sporozoites be killed [6]. The steepness of the curve in this region implies that incremental improvements in the proportion of parasites killed can lead to substantial improvements in efficacy against infection as the last few sporozoites are cleared up. An implication of this is that if two PE vaccines providing protection through different biological mechanisms are co-administered, efficacies are expected to combine synergistically. For example, if two vaccines kill $85 \%$ of sporozoites and prevent $20 \%$ of infections when administered on their own, they will kill $1-(1-0.85)^{2} \approx 98 \%$ of sporozoites when combined, but prevent approximately $60 \%$ of

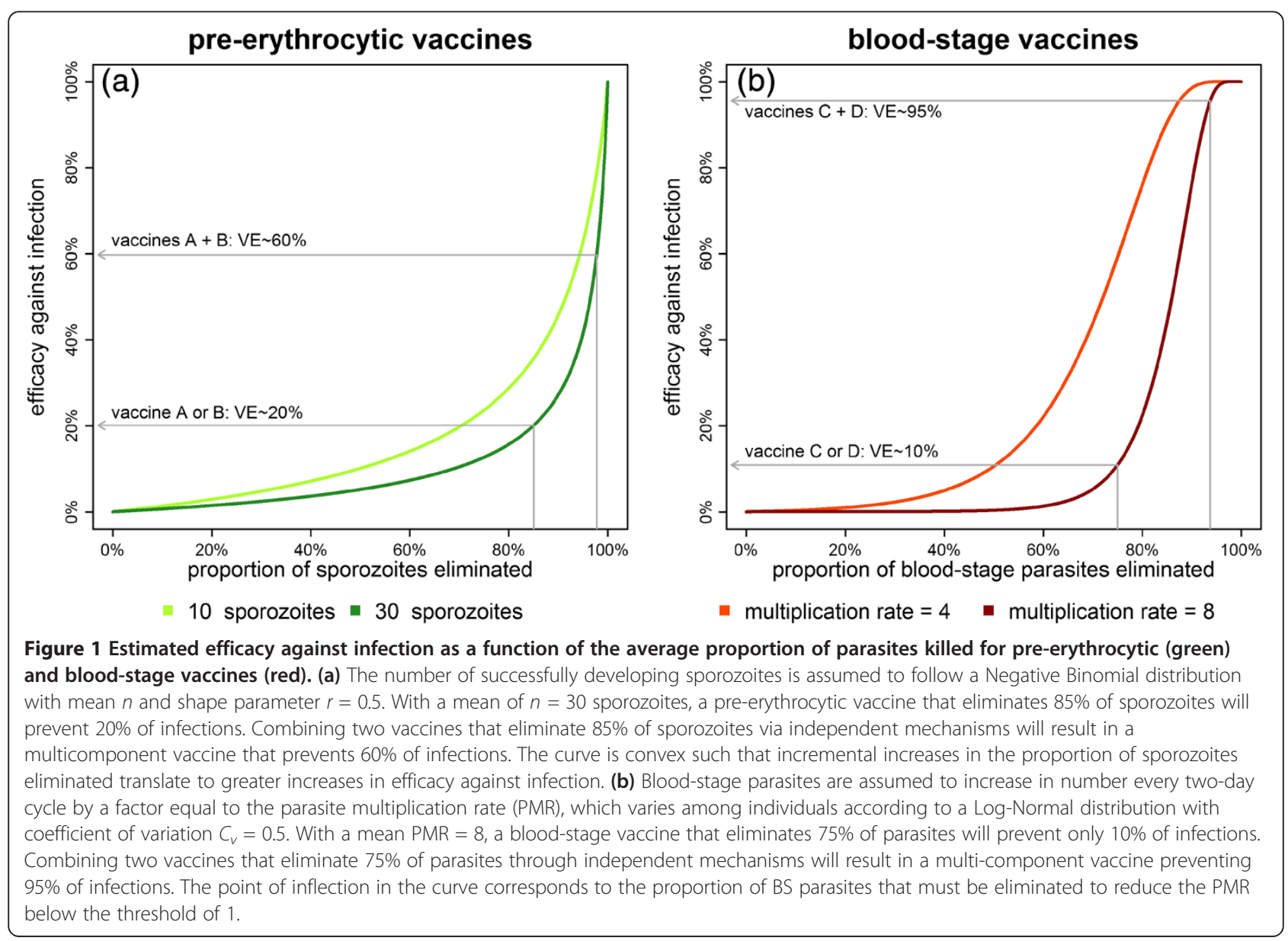


infections. There is also the potential for synergism for a combination of two BS vaccines, but this depends on whether the combined effect of the two vaccines is sufficient to reduce the PMR below the threshold of 1 in a large proportion of the vaccinated cohort. The threshold proportion of BS parasites killed to prevent infection corresponds to the inflection point of the curve in Figure $1 \mathrm{~b}$.

The challenge to vaccine developers in selecting an antigen to effectively target, may be turned into an opportunity to target multiple antigens [7] with efficacy combining synergistically. Synergy will depend not just on the initial efficacy after vaccination, but on the duration of each component vaccine, with waning driven by the vaccine with the shortest half-life.

\section{Combining existing vaccines may lead to synergistic improvements in efficacy}

RTS,S has been shown to prevent approximately $50 \%$ of infections by eliminating $>95 \%$ of sporozoites [8]. It is hypothesized that, due to the requirement for all sporozoites to be eliminated to prevent infection, combining RTS,S with a second vaccine that targets sporozoites via a different mechanism could lead to a multicomponent vaccine that prevents substantially more than $50 \%$ of infections. This would hold even if the second vaccine has negligible efficacy against infection when administered on its own, so long as it eliminates sufficient numbers of sporozoites [9], and there is no immunological interference between the two vaccines [10]. This phenomenon may also explain the high levels of efficacy against infection following immunization by irradiated sporozoites [11] which may confer protection by simultaneously inducing multiple, independent immune responses directed towards $P$. falciparum sporozoites.

\section{Competing interests}

The authors declare that they have no competing interests.

\section{Authors' contributions}

MTW and DLS contribute to the writing of the manuscript. Both authors read and approved the final manuscript.

\section{Acknowledgements}

Dr Sandy Douglas is thanked for helpful comments on the draft manuscript. MTW was funded by a Medical Research Council studentship and a grant from the Bell \& Melinda Gates Foundation.

\section{Author details}

${ }^{1}$ Department of Infectious Disease Epidemiology, MRC Centre for Outbreak Analysis and Modelling, Imperial College, London, UK. ²Department of Epidemiology, Johns Hopkins Bloomberg School of Public Health, Baltimore, Maryland, USA. ${ }^{3}$ Fogarty International Center, National Institutes of Health, Baltimore, Maryland, USA.

Received: 23 May 2013 Accepted: 30 July 2013

Published: 8 August 2013

\section{References}

1. Trieu A, Kayala MA, Burk C, Molina DM, Freilich DA, Richie TL, Baldi P, Felgner $\mathrm{PL}$, Doolan $\mathrm{DL}$ : Sterile protective immunity to malaria is associated with a panel of novel $P$. falciparum antigens. Molecul Cell Proteomics 2011, 10:M111.007948.

2. Douglas AD, Andrews L, Draper SJ, Bojang K, Milligan P, Gilbert SC, Imoukhuede EB, Hill AVS: Substantially reduced pre-patent parasite multiplication rates are associated with naturally acquired immunity to Plasmodium falciparum. J Inf Dis 2011, 203:1337-1340.

3. Medica DL, Sinnis P: Quantitative dynamics of Plasmodium yoelii sporozoite transmission by infected anopheline mosquitoes. Inf Immun 2005, 73:4363-4369.

4. Halloran ME, Longini IM, Struchiner CJ: Estimability and interpretation of vaccine efficacy using frailty mixing models. Am J Epidemiol 1996, 144:83-97.

5. RTS,S Vaccine Evaluation Group, Kester KE, Cummings JF, Ofori-Anyinam O, Ockenhouse CF, Krzych U, Moris P, Schwenk R, Nielsen RA, Debebe Z, Pinelis E, Juompan L, Williams J, Dowler M, Stewart VA, Wirtz RA, Dubois MC, Lievens M, Cohen J, Ballou WR, Heppner DG Jr: Randomized, double-blind, phase 2a trial of falciparum malaria vaccines RTS,S/AS01B and RTS,S/ AS02A in malaria-naive adults: safety, efficacy, and immunologic associates of protection. J Inf Dis 2009, 200:337-346.

6. Hill AVS: Pre-erythrocytic malaria vaccines: towards greater efficacy. Nature Rev Immunol 2006, 6:21-32

7. Saul A, Fay MP: Human immunity and the design of multi-component, single target vaccines. PLoS One 2007, 2:e850.

8. White MT, Bejon P, Olotu A, Griffin JT, Riley EM, Kester KE, Ockenhouse CF, Ghani AC: The relationship between RTS, S vaccine-induced antibodies, CD4(+) T cell responses and protection against Plasmodium falciparum infection. Plos One 2013, 8:e61395.

9. Bejon P, Andrews L, Andersen RF, Dunachie S, Webster D, Walther M, Gilbert SC, Peto T, Hill AVS: Calculation of liver-to-blood inocula, parasite growth rates, and preerythrocytic vaccine efficacy, from serial quantitative polymerase chain reaction studies of volunteers challenged with malaria sporozoites. J Inf Dis 2005, 191:619-626.

10. Heppner DG, Kester KE, Ockenhouse CF, Tornieporth N, Ofori O, Lyon JA, Stewart VA, Dubois P, Lanar DE, Krzych U, Moris P, Angov E, Cummings JF, Leach A, Hall BT, Dutta S, Schwenk R, Hillier C, Barbosa A, Ware LA, Nair L, Darko CA, Withers MR, Ogutu B, Polhemus ME, Fukuda M, Pichyangkul S, Gettyacamin M, Diggs C, Soisson L, Milman J, Dubois MC, Garçon N, Tucker K, Wittes J, Plowe CV, Thera MA, Duombo OK, Pau MG, Goudsmit J, Ballou WR, Cohen J: Towards an RTS, S-based, multi-stage, multi-antigen falciparum malaria: progress at the Walter Reed Army Institute of Research. Vaccine 2005, 23:2243-2250.

11. Hoffman SL, Goh LML, Luke TC, Schneider I, Le TP, Doolan DL, Sacci J, de la Vega P, Dowler M, Paul C, Gordon DM, Stoute JA, Church LW, Sedegah M, Heppner DG, Ballou WR, Richie TL: Protection of humans against malaria by immunization with radiation-attenuated Plasmodium falciparum sporozoites. J Inf Dis 2002, 185:1155-1164.

doi:10.1186/1475-2875-12-280

Cite this article as: White and Smith: Synergism from combinations of infection-blocking malaria vaccines. Malaria Journal 2013 12:280.

\section{Submit your next manuscript to BioMed Central and take full advantage of:}

- Convenient online submission

- Thorough peer review

- No space constraints or color figure charges

- Immediate publication on acceptance

- Inclusion in PubMed, CAS, Scopus and Google Scholar

- Research which is freely available for redistribution 